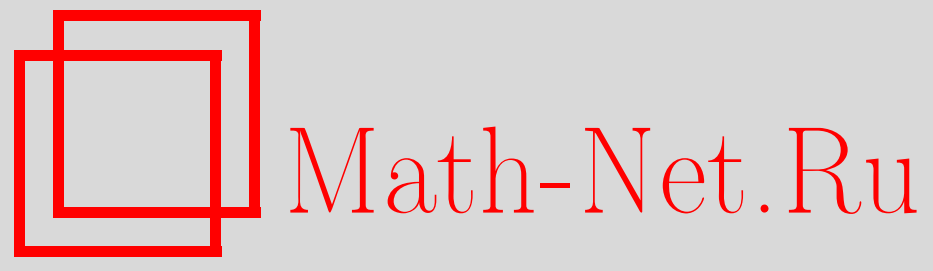

А. И. Днестрян, Об определении чистых состояний методом гомодинного детектирования, Вестн. Сам. гос. техн. ун-та. Сер. Физ.-мат. науки, 2016, номер 1, 33-42

DOI: https://doi.org/10.14498/vsgtu1462

Использование Общероссийского математического портала MathNet.Ru подразумевает, что вы прочитали и согласны с пользовательским соглашением

http://www . mathnet.ru/rus/agreement

Параметры загрузки:

IP : 35.174 .16 .151

26 апреля 2023 г., 12:24:05

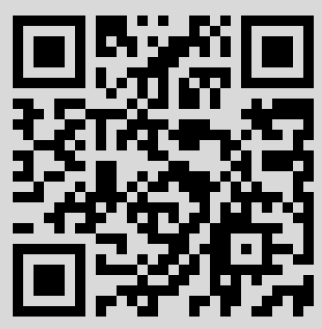


УДК 530.145.1

\title{
ОБ ОПРЕДЕЛЕНИИ ЧИСТЫХ СОСТОЯНИЙ МЕТОДОМ ГОМОДИННОГО ДЕТЕКТИРОВАНИЯ
}

\section{А. И. Днестрян}

Московский физико-технический институт (государственный университет), Россия, 141700, Московская обл., Долгопрудный, Институтский пер., 9.

\begin{abstract}
Аннотация
В работе обсуждаются методы реконструкции волновой функции чистого состояния квантовой системы по известной оптической томограмме состояния. Оптическая квантовая томограмма представляет собой однопараметрическое распределение вероятностей с параметром $\theta$. Волновая функция чистого состояния выражается через оптическую томограмму, если последняя известна для любых значений $\theta$. Однако оптическая томограмма определяется из эксперимента гомодинного детектирования, где $\theta$ фиксированно. Поэтому оптическая томограмма может быть известна лишь для нескольких дискретных значений параметра. Мы приводим приближенные методы определения волновой функции квантового состояния по неполной информации о его томограмме, представляющие собой развитие уже существующих методов.
\end{abstract}

Ключевые слова: квантовая томограмма, квантовое состояние, оператор плотности, волновая функция.

1. Введение. В конце XX века было предложено новое вероятностное представление квантовой механики, в которой квантовые состояния связываются со стандартными плотностями распределений вероятностей - так называемыми томографическими плотностями или квадратурными распределениями. Этот формализм, содержащий такую же информацию, как волновая функция и матрица плотности, основан на томографическом подходе к измерению квантовых состояний $[1,2]$. Суть его в следующем: каждому состоянию квантовой системы с матрицей плотности $\hat{\rho}$ ставится в соответствие плотность распределения наблюдаемой - квадратурной компоненты $\hat{X}=\mu \hat{q}+\nu \hat{p}$ :

$$
w(X, \mu, \nu)=\operatorname{Tr} \hat{\rho} \delta(X-\mu \hat{q}-\nu \hat{p})
$$

где $\hat{q}$ и $\hat{p}$ есть обычные операторы координаты и импульса. Функция (1) называется симплектической квантовой томограммой состояния $\hat{\rho}$. Частным случаем симплектической томограммы является экспериментально измеримая [2] оптическая томограмма

$$
w(X, \theta)=w(X, \mu=\cos \theta, \nu=\sin \theta) .
$$

(C) 2016 Самарский государственный технический университет.

\section{Образец для цитирования}

Днестрян А. И. Об определении чистых состояний методом гомодинного детектирования // Вестн. Сам. гос. техн. ун-та. Сер. Физ.-мат. науки, 2016. Т. 20, № 1. С. 33-42. doi: $10.14498 /$ vsgtu1462.

\section{Сведения об авторе}

Андрей Игоревич Днестрян (dnestor@inbox.ru), аспирант, каф. высшей математики. 
В работе [3] было установлено, что для чистого состояния с координатной волновой функцией $\psi(x)$ его симплектическая томограмма выражается следующим образом:

$$
w(X, \mu, \nu)=\left|\hat{F}_{\mu, \nu}[\psi]\right|^{2}(X),
$$

где $\hat{F}_{\mu, \nu}$ есть линейный интегральный оператор в $\mathscr{L}^{2}(\mathbb{R})$, подробно изученный в [4]. В данной статье мы рассмотрим вопрос обратимости отображения (2) в случае чистых состояний.

2. Реконструкция чистого состояния по его квадратурному распределению. Результатом всякой процедуры измерений над квантовой системой может быть только распределение вероятностей. Поскольку квантовое состояние содержит всю доступную информацию о квантовой системе, мы безусловно можем, отталкиваясь от этого состояния, рассчитать все распределения вероятностей [5]. Зададим обратный вопрос: возможно ли использовать набор вероятностных распределений для реконструкции квантового состояния?

Этот вопрос возвращает нас к раннему периоду развития квантовой механики, в частности, к обзорной статье В. Паули [6]. Он интересовался вопросом, можно ли найти амплитуду и фазу волновой функции, зная вероятности распределений по координате и импульсу. Паули не дал ответа на этот вопрос. Однако простые контрпримеры (см., например, [7-12]) показывают, что в общем случае это невозможно. На самом деле нужно знать больше распределений, чем эти два.

Как известно, функция Вигнера содержит всю информацию о квантовом состоянии, поэтому мы можем восстановить все квадратурные распределения с помощью преобразования Радона.

Преобразование вида

$$
w(x, \mu, \nu)=\int_{-\infty}^{\infty} \int_{-\infty}^{\infty} W(q, p) \delta(x-\mu q-\nu p) d q d p
$$

называется преобразованием Радона [13] функции $W(q, p)$. Функция (3) совпадает с симплектической томограммой [3]. Преобразование Радона обратимо, обращение преобразования (3) выглядит следующим образом:

$$
W(q, p)=\frac{1}{(2 \pi)^{2}} \int_{-\infty}^{\infty} \int_{-\infty}^{\infty} \int_{-\infty}^{\infty} w(x, \mu, \nu) e^{i(x-\mu q-\nu p)} d x d \mu d \nu .
$$

Обратимость преобразования Радона здесь позволяет выразить функцию Вигнера $W(q, p)$ через симплектическую томограмму состояния $w(x, \mu, \nu)$ при условии, что последняя известна для всех действительных $\mu, \nu$. Симплектическая томограмма обладает замечательным свойством однородности

$$
w(\lambda X, \lambda \mu, \lambda \nu)=\frac{1}{|\lambda|} w(X, \mu, \nu)
$$

используя которое, можно восстановить функцию Вигнера, зная лишь оптическую томограмму $w(x, \theta)$ для любого $\theta \in[0, \pi][14,15]$. В этом случае функция Вигнера выражается по формуле

$$
W(q, p)=\frac{1}{(2 \pi)^{2}} \int_{0}^{\pi} d \theta \int_{-\infty}^{+\infty}|t| d t \int_{-\infty}^{+\infty} w(x, \theta) \exp i t(x-q \cos \theta-p \sin \theta) d x .
$$


Однако реально невозможно измерить оптическую томограмму (квадратурное распределение) $w(x, \theta)$ для любы з значений фазы $\theta$. Возможно лишь провести гомодинное детектирование для различных, но фиксированных значений фазы $\theta$. На практике, когда такой подход реализуется, получается ансамбль распределений $\left\{w\left(x, \theta_{1}\right), w\left(x, \theta_{2}\right), \ldots, w\left(x, \theta_{N}\right)\right\}$, который с некоторой погрешностью можно считать за истинный непрерывный ансамбль. Затем по этому ансамблю численно с помощью обратного преобразования Радона восстанавливается функция Вигнера исследуемого состояния.

В эксперименте фазу $\theta$ изменяют, меняя разность хода поступающих на светоделитель лучей. Удобно выбирать значения фаз эквидистантными $\theta_{n}=$ $=\pi(n-1) / N$. Поэтому для осуществления обратного преобразования Радона необходимо, чтобы $n$ пробегало значения от 1 до $N$. Ясно, что точность вычислений увеличивается с ростом $N$, т. к. численное интегрирование представляет собой суммирование с шагом $h \propto N^{-1}$. Однако зачастую проведение экспериментов со значениями $N \geqslant 10$ бывает затруднительным.

Вместе с этим количество фаз $N$ еще и качественно влияет на результат. В работе [16] было показано, что для точной реконструкции состояния в оптической гомодинной томографии размерность матрицы плотности в представлении Фока должна быть равна количеству фаз $\theta_{n}$, для которых было произведено гомодинное детектирование. Также был получен простой способ оценки ошибок, если фактическая размерность матрицы плотности больше, чем число фаз, используемых в эксперименте.

Еще один метод реконструкции волновой функции состояния был дан в [17]. Он основан на интегральных представлениях коэффициентов в разложении волновой функции. Развивая идею, высказанную авторами, мы предлагаем следующий метод.

Пусть произведен эксперимент гомодинного детектирования некого состояния. На выходе эксперимента для фиксированного значения фазы $\theta$ мы имеем относительно большой $\left(\sim 10^{5}\right)$ набор точек $x_{j}$ - измеренных квадратурных компонент, по которому строится гистограмма. Эта гистограмма определяет распределение $w(x, \theta)$. Предположим, что чистое состояние

$$
\hat{\rho}=|\psi\rangle\langle\psi|,
$$

подаваемое на гомодинный детектор, представимо конечной суммой фоковских состояний, т. е. волновая функция может быть представлена в виде

$$
|\psi\rangle=\sum_{n=0}^{N} c_{n}|n\rangle
$$

где

$$
\langle x \mid n\rangle=\frac{1}{\pi^{1 / 4} \sqrt{2^{n} n !}} H_{n}(x) e^{-x^{2} / 2} .
$$

Фоковские состояния образуют ортонормированный базис

$$
\langle n \mid m\rangle=\delta_{n m},
$$

поэтому коэффициенты $c_{n}$ удовлетворяют

$$
\sum_{n=0}^{N}\left|c_{n}\right|^{2}=1
$$


Обозначая $\langle x \mid n\rangle=\psi_{n}(x)$ и подставляя разложение (4) в равенство (2), мы получаем оптическую томограмму суперпозиции фоковских состояний [4]

$$
\begin{aligned}
& w(x, \theta)=\left|\hat{F}_{\cos \theta, \sin \theta}[\psi]\right|^{2}=\left|\sum_{n=0}^{N} c_{n} \psi_{n}(x) e^{-i n \theta}\right|^{2}= \\
& =\sum_{n, m \geqslant 0}^{N} \operatorname{Re}\left(c_{n} c_{m}^{*} e^{i \theta(m-n)}\right) \psi_{n}(x) \psi_{m}(x)= \\
& =\sum_{n=0}^{N}\left|c_{n}\right|^{2} \psi_{n}^{2}(x)+2 \sum_{0 \leqslant m<l \leqslant N} \operatorname{Re}\left(c_{m} c_{l}^{*} e^{i \theta(l-m)}\right) \psi_{m}(x) \psi_{l}(x)= \\
& =\sum_{n, m \geqslant 0}^{N} c_{n} c_{m}^{*} e^{i \theta(m-n)} \psi_{n}(x) \psi_{m}(x)
\end{aligned}
$$

или через амплитуду и фазу коэффициентов $c_{n}=\left|c_{n}\right| e^{i \varphi_{n}}$ :

$$
w(x, \theta)=\sum_{0 \leqslant n, m \leqslant N}\left|c_{n}\right|\left|c_{m}\right| \cos \left(\theta(m-n)+\left(\varphi_{n}-\varphi_{m}\right)\right) \psi_{n}(x) \psi_{m}(x) .
$$

Произведение волновых функций фоковских состояний $\psi_{n}(x) \psi_{m}(x)$ раскладывается в сумму [17]

$$
\psi_{n}(x) \psi_{m}(x)=2^{1 / 4} \sum_{k=0}^{n+m} \beta_{k}^{n, m} \psi_{k}(\sqrt{2} x),
$$

где коэффициенты $\beta_{k}^{n, m}$ не равны нулю, только если числа $n+m$ и $k$ одной четности, при этом для незануляющихся коэффициентов

$$
\beta_{k}^{n, m}=\left(\frac{2}{\pi}\right)^{1 / 4} \sqrt{\frac{n ! m !}{k !}} 2^{-2 q-(k+1) / 2}(-1)^{q} \sum_{j=0}^{\min (n, m, q)} \frac{(-4)^{j}(n+m-2 j) !}{j !(n-j) !(m-j) !(q-j) !},
$$

где $q=(n+m-k) / 2$. В таблице представлены коэффициенты $\beta_{k}^{n, m}$ для $1 \leqslant n \leqslant 2,1 \leqslant m \leqslant 5$, определенные численными методами по формуле

$$
\beta_{k}^{n, m}=2^{1 / 4} \int_{-\infty}^{+\infty} \psi_{n}(x) \psi_{m}(x) \psi_{k}(\sqrt{2} x) d x
$$

следующей напрямую из (8). Отсюда следует, что (7) можно переписать так:

$$
w(x, \theta)=2^{1 / 4} \sum_{0 \leqslant n, m \leqslant N} \sum_{k=0}^{n+m}\left|c_{n}\right|\left|c_{m}\right| \cos \left(\theta(m-n)+\left(\varphi_{n}-\varphi_{m}\right)\right) \beta_{k}^{n, m} \psi_{k}(\sqrt{2} x) .
$$

Поскольку $\psi_{k}(\sqrt{2} x)$ образуют ортогональный базис в $\mathscr{L}^{2}(\mathbb{R})$, равенство $(10)$ представляет из себя разложение $w(x, \theta)$ в ряд Фурье, а коэффициенты этого ряда находятся из условия 


$$
\begin{aligned}
\sum_{0 \leqslant n, m \leqslant N}\left|c_{n}\right|\left|c_{m}\right| \cos \left(\theta(m-n)+\left(\varphi_{n}-\varphi_{m}\right)\right) & \beta_{k}^{n, m}= \\
& =2^{1 / 4}\left(w(x, \theta), \psi_{k}(\sqrt{2} x)\right) .
\end{aligned}
$$

Скалярное произведение в правой части $(11)$ определено в $\mathscr{L}^{2}(\mathbb{R})$, т. е.

$$
\left(w(x, \theta), \psi_{k}(\sqrt{2} x)\right)=\int_{-\infty}^{+\infty} w(x, \theta) \psi_{k}(\sqrt{2} x) d x .
$$

Индекс $k$ в (11) пробегает $2 N+1$ значений: $0 \leqslant k \leqslant 2 N$. Тем самым для нахождения коэффициентов $c_{n}$ разложения (4) мы имеем систему из $2 N+1$ уравнений, повторяющих (11) для разных значений $k$ :

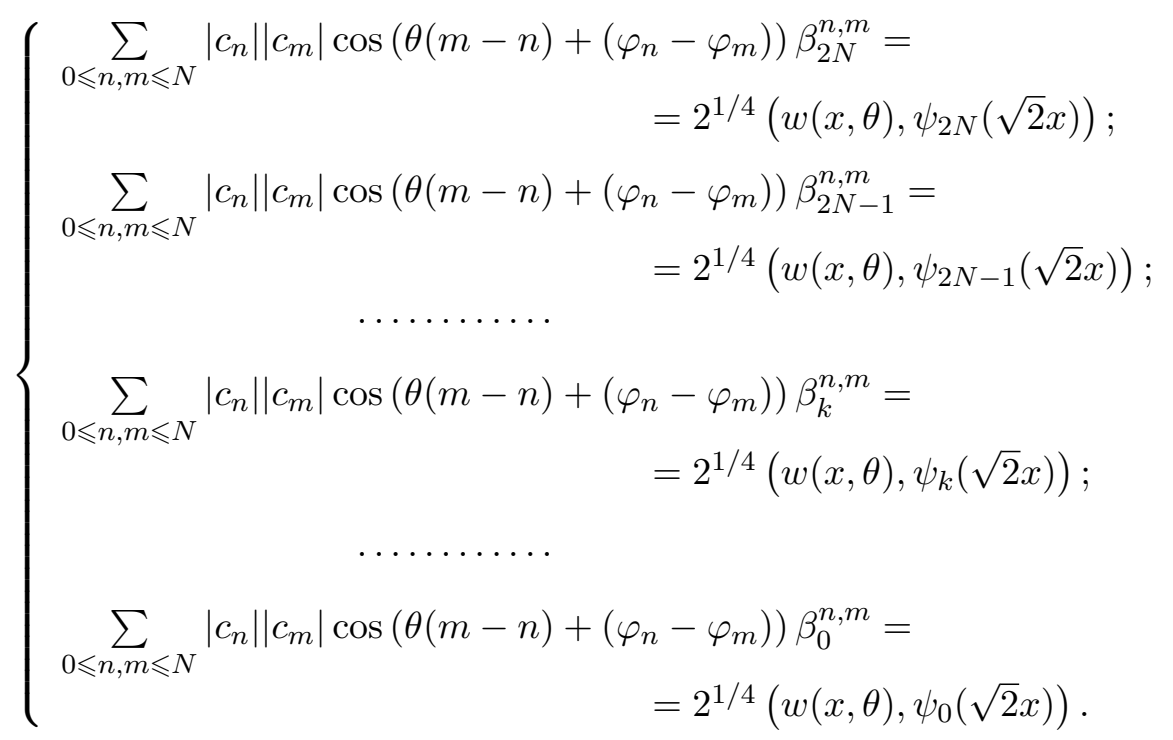

Система уравнений (12), вообще говоря, содержит $2 N+2$ неизвестных $\left(\left\{c_{k}\right\}_{k=0}^{N}\right.$ и $\left.\left\{\varphi_{k}\right\}_{k=0}^{N}\right)$ и $2 N+1$ уравнений, однако она не является недоопределенной. Дело в том, что искомая волновая функция $\psi(x)$ в любом случае может быть определена с точностью до постоянной фазы. По этой причине мы фиксируем (зануляем) фазу, например, коэффициента $c_{N}$, а все остальные фазы считаем относительно фазы $\varphi_{N}=0$. После этого предположения число неизвестных системы (12) становится равным $2 N+1$.

На первый взгляд, решение системы уравнений (12) представляется очень трудным процессом, однако есть один момент, заметно упрощающий ее решение. Суть в следующем: коэффиценты $\beta_{k}^{n, m}=0$ при $n+m<k$. Следовательно, первые уравнения перепишутся заметно проще:

$$
\left\{\begin{array}{c}
c_{N}^{2} \beta_{2 N}^{N, N}=2^{1 / 4}\left(w(x, \theta), \psi_{2 N}(\sqrt{2} x)\right) \\
2 c_{N} c_{N-1} \cos \left(\theta-\varphi_{N-1}\right) \beta_{2 N-1}^{N, N-1}=2^{1 / 4}\left(w(x, \theta), \psi_{2 N-1}(\sqrt{2} x)\right) \\
\ldots \ldots \ldots \cdots
\end{array}\right.
$$

Отсюда видно, что $c_{N}$ находится из первого уравнения, а $c_{N-1}$ выражается через $c_{N}$ и т. д. Таким образом, данная методика позволяет определить 
Таблица коэффициентов $\boldsymbol{\beta}_{\boldsymbol{k}}^{\boldsymbol{n}, \boldsymbol{m}}$, вычисленных по (9). Верхняя таблица содержит коэффициенты $\beta_{k}^{1, m}$, т. е. при $n=1$, нижняя таблица - при $n=2$ [The table contains the coefficients $\beta_{k}^{n, m}$ calculated by the Eq. (9). The upper part of the table contains the coefficients $\boldsymbol{\beta}_{\boldsymbol{k}}^{\mathbf{1}, \boldsymbol{m}}$; The lower part of the table contains the coefficients $\beta_{k}^{2, m}$ ]

\begin{tabular}{|c||c|c|c|c|c|}
\hline$n=1$ & $m=1$ & $m=2$ & $m=3$ & $m=4$ & $m=5$ \\
\hline \hline$k=1$ & 0 & 0.1579 & 0 & -0.2051 & 0 \\
\hline$k=2$ & 0.4466 & 0 & 0 & 0 & -0.1528 \\
\hline$k=3$ & 0 & 0.3868 & 0 & -0.1116 & 0 \\
\hline$k=4$ & 0 & 0 & 0.3158 & 0 & -0.1765 \\
\hline$k=5$ & 0 & 0 & 0 & 0.2496 & 0 \\
\hline$k=6$ & 0 & 0 & 0 & 0 & 0.1934 \\
\hline \hline$n=2$ & $m=1$ & $m=2$ & $m=3$ & $m=4$ & $m=5$ \\
\hline \hline$k=1$ & 0.1579 & 0 & 0.0967 & 0 & -0.1621 \\
\hline$k=2$ & 0 & 0.2233 & 0 & -0.0483 & 0 \\
\hline$k=3$ & 0.3867 & 0 & 0.1579 & 0 & -0.1324 \\
\hline$k=4$ & 0 & 0.3867 & 0 & -0.0558 & 0 \\
\hline$k=5$ & 0 & 0 & 0.3531 & 0 & -0.0395 \\
\hline$k=6$ & 0 & 0 & 0 & 0.3058 & 0 \\
\hline$k=7$ & 0 & 0 & 0 & 0 & 0.2558 \\
\hline
\end{tabular}

состояние $\psi(x)$ в представлении (4), т. е. в виде суперпозиции первых $N$ фоковских состояний. Отметим, что исходный метод [17] решает данную задачу при $N=2$.

Перейдем к следующему методу реконструкции волновой функции, который является развитием метода, представленного в [18]. Вводя коэффициент

$$
\alpha_{n}=\frac{1}{\sqrt[4]{\pi} \sqrt{2^{n} n !}}
$$

перепишем равенство (6), используя (5):

$$
\begin{aligned}
& w(x, \theta)=\sum_{n=0}^{N} \alpha_{n}^{2}\left|c_{n}\right|^{2} H_{n}^{2}(x) e^{-x^{2}}+ \\
& \quad+2 \sum_{0 \leqslant m<l \leqslant N} \alpha_{m} \alpha_{l} \operatorname{Re}\left(c_{m} c_{l}^{*} e^{i \theta(l-m)}\right) H_{m}(x) H_{l}(x) e^{-x^{2}} .
\end{aligned}
$$

Домножим обе части этого равенства на $e^{x^{2}}$ и получим

$$
e^{x^{2}} w(x, \theta)=\sum_{n=0}^{N} \alpha_{n}^{2}\left|c_{n}\right|^{2} H_{n}^{2}(x)+2 \sum_{0 \leqslant m<l \leqslant N} \alpha_{m} \alpha_{l} \operatorname{Re}\left(c_{m} c_{l}^{*} e^{i \theta(l-m)}\right) H_{m}(x) H_{l}(x) .
$$

В этом равенстве справа стоит многочлен степени $2 N$, его коэффициенты 
можно определить, дифференцируя этот многочлен пошагово:

$$
\left\{\begin{aligned}
\frac{d^{2 N}}{d x^{2 N}}\left(e^{x^{2}} w(x, \theta)\right)= & \alpha_{N}^{2}(2 N) ! 2^{2 N}\left|c_{N}\right|^{2} \\
\frac{d^{2 N-1}}{d x^{2 N-1}}\left(e^{x^{2}} w(x, \theta)\right)= & \alpha_{N}^{2}(2 N) ! 2^{2 N}\left|c_{N}\right|^{2} x+ \\
& +2 \alpha_{N}^{2} N(N-1) 2^{N-2} 2^{N}(2 N-1) !\left|c_{N}\right|^{2}+ \\
& +2^{N} 2^{N-1}(2 N-1) ! \alpha_{N} \alpha_{N-1} \operatorname{Re}\left(c_{N} c_{N-1}^{*} e^{i \theta}\right) \\
& \ldots
\end{aligned}\right.
$$

Система содержит $2 N+1$ уравнений и $2 N+2$ неизвестных, доопределим эту систему условием нормировки волновой функции

$$
\sum_{n=0}^{N}\left|c_{n}\right|^{2}=1
$$

Тогда количество неизвестных и уравнений совпадает и равно $2 N+2$. Решение системы дает коэффициенты $c_{n}$ в разложении (4).

3. Заключение. В данной статье развиты уже существующие методы реконструкции чистого квантового состояния по неполной информации о его квантовой томограмме. Состояние описывается волновой функцией, которая в рамках данной работы аппроксимируется суммой из $N$ фоковских состояний. Отметим, что по сравнению с начальными методами, где $N$ ограничено, в данной работе это число произвольно. С ростом количества слагаемых данной суммы в силу сходимости можно добиться сколь угодно большой точности приближений волновой функции. Также метод, развитый в данной работе, имеет преимущество перед обратным преобразованием Радона, так как последнее осуществимо только если известны квадратурные распределения $w(x, \theta)$ на сетке $\theta_{n}$, покрывающей отрезок $[0 ; \pi]$, тогда как метод, предложенный в работе, требует, чтобы было известно квадратурное распределение лишь для одного значения $\theta$. Это преимущество существенно при проведении экспериментов гомодинного детектирования.

Отметим, что на практике всегда может быть известна лишь неполная информация о томограммме, поскольку томограмма является экспериментально наблюдаемой величиной, и результат эксперимента представляет собой набор значений томограммы в разных точках. Этот факт обуславливает актуальность задачи, рассмотренной в данной работе.

\section{ORCID}

Андрей Игоревич Днестрян: http://orcid.org/0000-0002-9381-2133 


\section{БИБЛИОГРАФИЧЕСКИЙ СПИСОК}

1. Vogel K., Risken H. Determination of quasiprobability distributions in terms of probability distributions for the rotated quadrature phase // Phys. Rev. A, 1989. vol. 40, no. 5. pp. $2847-$ 2855. doi: 10.1103/physreva.40.2847.

2. Smithey D. T., Beck M., Raymer M. G., Faridani A. Measurement of the Wigner distribution and the density matrix of a light mode using optical homodyne tomography: Application to squeezed states and the vacuum // Phys. Rev. Lett., 1993. vol.70, no. 9. pp. 1244-1247. doi: $10.1103 /$ physrevlett.70.1244.

3. Mancini S., Man'ko V. I., Tombesi P. Symplectic tomography as classical approach to quantum systems // Phys. Lett. A, 1996. vol.213, no.1-2. pp. 1-6. doi:10.1016/ 0375-9601 (96)00107-7.

4. Амосов Г. Г., Днестрян А. И. О спектре семейства интегральных операторов, определяющих квантовую томограмму // Труды МФТИ, 2011. Т. 3, № 1. С. 5-9.

5. Schleich W. P. Quantum Optics in Phase Space. Berlin: Verlag, 2001, xx+695 pp. doi: 10 . 1002/3527602976.

6. Pauli W. Die allgemeinen Prinzipien der Wellenmechanik // Handbuch Physik, 1933. vol. 24, Tl. 1. pp. 83-272; Pauli W. Die allgemeinen Prinzipien der Wellenmechanik / Die allgemeinen Prinzipien der Wellenmechanik / Neu herausgegeben und mit historischen Anmerkungen versehen von Norbert Straumann; ed. Professor Dr. Norbert Straumann. Berlin: Springer, 1990. pp. 21-192. doi : 10.1007/978-3-642-61287-9_2.

7. Reichenbach H. Philosophic Foundations of Quantum Mechanics. Berkeley and Los Angeles: University of California Press, 1944. x+182 pp.

8. Vogt A. Position and Momentum Distributions do not Determine the Quantum Mechanical State / Mathematical Foundations of Quantum Theory. New York: Academic Press, 1978. pp. 365-372. doi : 10.1016/b978-0-12-473250-6.50024-8.

9. Freyberger M., Bardroff P. J., Leichle C., Schrade G., Schleich W. P. The art of measuring quantum states // Physics World, 1997. vol. 10, no. 11. pp. 41-46. doi: 10.1088/2058-7058/ 10/11/31.

10. Schleich W. P., Raymer M. G. Special issue on quantum state preparation and measurement // J. Mod. Opt., 1997. no. 11-12. pp. 2021-2022. doi: 10.1080/09500349708231863.

11. Leibfried D., Pfau T., Monroe C. Shadows and Mirrors: Reconstructing Quantum States of Atom Motion // Physics Today, 1998. vol.51, no.4. pp. 22-28. doi: 10.1063/1.882256.

12. Welsch D.-G., Vogel W., Opatrný T. II Homodyne Detection and Quantum-State Reconstruction / Progress in Optics. vol. 39; ed. E. Wolf. Amsterdam: North-Holland, 1999. pp. 63-211. doi: 10.1016/S0079-6638(08)70389-5.

13. Radon J. Über die Bestimmung von Funktionen durch ihre Integralwerte längs gewisser Mannigfaltigkeiten// Ber. Verh. Sächs. Akad. Wiss. Leipzig, Math. Nat. kl., 1917. vol. 69. pp. 262-277, Available at http://people.csail.mit.edu/bkph/courses/papers/Exact_ Conebeam/Radon_Deutsch_1917.pdf (February 24, 2016); Radon J. Über die Bestimmung von Funktionen durch ihre Integralwerte längs gewisser Mannigfaltigkeiten / Proceedings of Symposia in Applied Mathematics. vol. 27; ed. Lawrence A. Shepp. Providence: Amer. Math. Soc., 1983, pp. 71-86. doi: 10.1090/psapm/027/692055.

14. D'Ariano G. M., Mancini S., Man'ko V. I., Tombesi P. Reconstructing the density operator by using generalized field quadratures // Quantum Semiclass. Opt., 1996. vol.8, no. 5. pp. 1017-1027. doi: 10.1088/1355-5111/8/5/007.

15. Leonhardt U., Paul H., D'Ariano G. M. Tomographic reconstruction of the density matrix via pattern functions // Phys. Rev. A, 1995. vol.52, no.6. pp. 4899-4907. doi: 10.1103/ physreva.52.4899.

16. Leonhardt U., Munroe M. Number of phases required to determine a quantum state in optical homodyne tomography // Phys. Rev. A, 1996. vol. 54, no. 4. pp. 3682-3684. doi: 10. 1103/physreva.54.3682.

17. Orłowski A., Paul H. Phase retrieval in quantum mechanics // Phys. Rev. A, 1994. vol. 50, no. 2. pp. R921-R924. doi : 10.1103/physreva.50.r921. 
18. Амосов Г. Г., Днестрян А. И. О восстановлении чистого состояния по неполной информации о его оптической томограмме // Изв. вузов. Матем., 2013. № 3. С. 62-67.

Поступила в редакцию $21 / \mathrm{XI} / 2015$;

в окончательном варианте - 24/II/2016;

принята в печать $-26 / \mathrm{II} / 2016$.

Vestn. Samar. Gos. Techn. Un-ta. Ser. Fiz.-mat. nauki

[J. Samara State Tech. Univ., Ser. Phys. \& Math. Sci.], 2016, vol. 20, no. 1, pp. 33-42

ISSN: 2310-7081 (online), 1991-8615 (print)

doi: http://dx.doi.org/10.14498/vsgtu1462

\title{
MSC: $81 P 50$
}

\section{ON THE DETERMINATION OF PURE QUANTUM STATES BY THE HOMODYNE DETECTION}

\section{A. I. Dnestryan}

Moscow Institute of Physics and Technology (State University),

9, Inststitutskii per., Dolgoprudny, Moscow region, 141700, Russian Federation.

\begin{abstract}
The methods of reconstruction of the wave function of a pure state of a quantum system by quadrature distribution measured experimentally by the homodyne detection are considered. Such distribution is called optical tomogram of a state and containes one parameter $\theta$. Wave function of a state is determined exactly by its optical tomogram if last one is known for all $\theta$. But one can obtain optical tomogram from experiment of homodyne detection only for discrete number of $\theta$. We introduce some approximate methods of reconstructing the state by such information about its optical tomogram.
\end{abstract}

Keywords: quantum tomography, quantum state, density operator, wave function.

\section{ORCID}

Andrey I. Dnestryan: http://orcid.org/0000-0002-9381-2133

\section{REFERENCES}

1. Vogel K., Risken H. Determination of quasiprobability distributions in terms of probability distributions for the rotated quadrature phase, Phys. Rev. A, 1989, vol.40, no. 5, pp. 28472855. doi: 10.1103/physreva.40.2847.

2. Smithey D. T., Beck M., Raymer M. G., Faridani A. Measurement of the Wigner distribution and the density matrix of a light mode using optical homodyne tomography: Application to squeezed states and the vacuum, Phys. Rev. Lett., 1993, vol.70, no. 9, pp. 1244-1247. doi: $10.1103 /$ physrevlett.70.1244.

(C) 2016 Samara State Technical University.

\section{Please cite this article in press as:}

Dnestryan A. I. On the determination of pure quantum states by the homodyne detection, Vestn. Samar. Gos. Tekhn. Univ., Ser. Fiz.-Mat. Nauki [J. Samara State Tech. Univ., Ser. Phys. \& Math. Sci.], 2016, vol. 20, no. 1, pp. 33-42. doi: 10.14498/vsgtu1462. (In Russian)

Author Details:

Andrey I. Dnestryan (dnestor@inbox.ru), Postgraduate Student, Dept. of Higher Mathematics. 
3. Mancini S., Man'ko V. I., Tombesi P. Symplectic tomography as classical approach to quantum systems, Phys. Lett. A, 1996, vol.213, no.1-2, pp. 1-6. doi:10.1016/ 0375-9601 (96) 00107-7.

4. Amosov G. G., Dnestryan A. I. On the spectrum of a family of integral operators defining the quantum tomogram, Trudy MFTI, 2011, vol. 3, no. 1, pp. 5-9 (In Russian).

5. Schleich W. P. Quantum Optics in Phase Space. Berlin, Verlag, 2001, xx+695 pp. doi: 10 . 1002/3527602976.

6. Pauli W. Die allgemeinen Prinzipien der Wellenmechanik, Handbuch Physik, 1933, vol. 24, Tl. 1, pp. 83-272; Pauli W. Die allgemeinen Prinzipien der Wellenmechanik, Die allgemeinen Prinzipien der Wellenmechanik, Neu herausgegeben und mit historischen Anmerkungen versehen von Norbert Straumann; ed. Professor Dr. Norbert Straumann. Berlin, Springer, 1990. pp. 21-192. doi: 10.1007/978-3-642-61287-9_2.

7. Reichenbach H. Philosophic Foundations of Quantum Mechanics. Berkeley and Los Angeles, University of California Press, 1944, x+182 pp.

8. Vogt A. Position and Momentum Distributions do not Determine the Quantum Mechanical State, Mathematical Foundations of Quantum Theory. New York, Academic Press, 1978, pp. 365-372. doi : 10.1016/b978-0-12-473250-6.50024-8.

9. Freyberger M., Bardroff P. J., Leichle C., Schrade G., Schleich W. P. The art of measuring quantum states, Physics World, 1997, vol.10, no.11, pp. 41-46. doi: 10.1088/2058-7058/ $10 / 11 / 31$.

10. Schleich W. P., Raymer M. G. Special issue on quantum state preparation and measurement, J. Mod. Opt., 1997, no. 11-12, pp. 2021-2022. doi: 10.1080/09500349708231863.

11. Leibfried D., Pfau T., Monroe C. Shadows and Mirrors: Reconstructing Quantum States of Atom Motion, Physics Today, 1998, vol.51, no.4, pp. 22-28. doi: 10.1063/1.882256.

12. Welsch D.-G., Vogel W., Opatrný T. II Homodyne Detection and Quantum-State Reconstruction, Progress in Optics, vol. 39; ed. E. Wolf. Amsterdam, North-Holland, 1999, pp. 63-211. doi: 10.1016/S0079-6638(08)70389-5.

13. Radon J. Über die Bestimmung von Funktionen durch ihre Integralwerte längs gewisser Mannigfaltigkeiten, Ber. Verh. Sächs. Akad. Wiss. Leipzig, Math. Nat. kl., 1917, vol.69, pp. 262-277, Available at http://people.csail.mit.edu/bkph/courses/papers/Exact_ Conebeam/Radon_Deutsch_1917.pdf (February 24, 2016); Radon J. Über die Bestimmung von Funktionen durch ihre Integralwerte längs gewisser Mannigfaltigkeiten, Proceedings of Symposia in Applied Mathematics. vol. 27; ed. Lawrence A. Shepp. Providence, Amer. Math. Soc., 1983, pp. 71-86. doi : 10.1090/psapm/027/692055.

14. D'Ariano G. M., Mancini S., Man'ko V. I., Tombesi P. Reconstructing the density operator by using generalized field quadratures, Quantum Semiclass. Opt., 1996, vol.8, no.5, pp. 1017-1027. doi : 10.1088/1355-5111/8/5/007.

15. Leonhardt U., Paul H., D'Ariano G. M. Tomographic reconstruction of the density matrix via pattern functions, Phys. Rev. A, 1995, vol.52, no.6, pp. 4899-4907. doi: 10.1103/ physreva.52.4899.

16. Leonhardt U., Munroe M. Number of phases required to determine a quantum state in optical homodyne tomography, Phys. Rev. A, 1996, vol.54, no.4, pp. 3682-3684. doi: 10. 1103/physreva.54.3682.

17. Orłowski A., Paul H. Phase retrieval in quantum mechanics, Phys. Rev. A, 1994, vol.50, no. 2, pp. R921-R924. doi: 10.1103/physreva.50.r921.

18. Amosov G. G., Dnestryan A. I. Reconstruction of a pure state from incomplete information on its optical tomogram, Russian Math. (Iz. VUZ), 2013, vol. 57, no. 3, pp. 51-55. doi : 10 . 3103/S1066369X13030079.

Received 21/XI/2015; received in revised form $24 / \mathrm{II} / 2016$; accepted 26/II/2016. 